\title{
The motion of a plate in a rotating fluid at an arbitrary angle of attack
}

\author{
By DAVID C. WILCOX \\ California Institute of Technology†
}

(Received 23 September 1971 and in revised form 3 April 1972)

Slow motion of a thin plate at a finite angle of attack in a rotating container filled with a viscous incompressible fluid is analysed. The Rossby and Ekman numbers are assumed to be small. The solution method is developed by studying horizontal translation of an elliptical plate. The plate is shown to carry a stagnant Taylor column with it as it moves. Detailed analysis of the structure of the vertical shear column bounding the Taylor column is circumvented by integrating the equations of motion across the shear column. A jump condition based upon mass conservation in the shear column which relates the geostrophic regions inside and outside the Taylor column results. This jump condition and its method of derivation can be used to analyse arbitrary (slow) motion of any thin plate at any angle of attack.

The fluid motion resulting when a disk moves using all six degrees of freedom at an infinitesimal angle of attack is discussed. The forces and moments on the disk are calculated and the streamlines of the geostrophic flow are displayed.

\section{Introduction}

One of the most interesting phenomena of rotating-fluid dynamics is the Taylor column. In his original study of horizontal (normal to the sense of rotation) motion of a sphere, Taylor (1922) found that the fluid inside the cylindrical column above and below the sphere was motionless relative to the body. In a subsequent study involving horizontal motion of bodies whose thickness is large relative to the Ekman-layer thickness, Jacobs (1964) also found the Taylor column to be stagnant. The fluid in the Taylor column does not always move with the same velocity as the body, however, even for horizontal motion. Examples are provided by Moore \& Saffman $(1969 a, b)$, who analysed the fluid motion ensuing from vertical and horizontal translation of a disk in a container bounded by horizontal walls.

For vertical translation of the disk (assumed to lie in a plane parallel to the container walls) the vertical velocity of the fluid inside the Taylor column was found to be half the disk velocity (Moore \& Saffman 1969a). The geostrophic flow was shown to be determined uniquely by the Ekman compatibility relation. There is a detached shear column separating the geostrophic regions inside and outside the Taylor column. The shear column, which must be inserted to make the

† Present address: Applied Theory, Inc., 1010 Westwood Blvd., Los Angeles, California 90024. 
solution analytic across the boundary of the Taylor column and to complete the circulation of the fluid between the walls and the disk, therefore plays a passive role in determining the geostrophic flow. For horizontal translation, the fluid passes through the Taylor column and is deflected by $18.4^{\circ}$ from the direction of translation. However, Moore \& Saffman (1969b) demonstrated that the Ekman condition is not sufficient to specify the solution in the geostrophic region for this case. Appeal to the dynamics of the shear column was made to remove the indeterminateness of the geostrophic flow. Hence, the shear column plays an active role for this motion.

An additional complication is introduced if the disk, or, more generally, a thin plate, is inclined to the walls at a finite angle $\alpha$. There is an extra degree of indeterminateness associated with an apparent absence of irrotationality of the geostrophic flow in the regions between the plate and the walls of the container where the geostrophic contours are not closed. This is the problem addressed in this study. The geostrophic flow is found to be indeterminate and the shear column active for any (slow) plate motion. The techniques first suggested by Stewartson (1966) and later exploited by Moore \& Saffman for analysing the shear column are employed although their implementation is more subtle here than in previous problems.

Horizontal translation of an elliptical plate for finite $\alpha$ is analysed in $\S 2$. A jump condition relating the geostrophic regions on each side of the detached shear column is developed. The limiting form of the jump condition for $\alpha \ll 1$ is shown in $\S 3$. Slow motion of a disk using all six degrees of freedom is also described for an infinitesimal angle of attack.

\section{Motion of a plate at a finite angle of attack}

In this section, the fluid motion resulting from translation of an elliptical plate in a horizontal direction is examined in detail. The results of the analysis are then generalized and cast in a form applicable to arbitrary slow motion of any thin plate.

\subsection{Statement of the problem}

A container filled with a viscous incompressible fluid of kinematic viscosity $\nu$ rotates at angular velocity $\Omega$ about what will be termed its vertical axis. The container is bounded above and below by horizontal walls. Between the walls there is a thin plate of characteristic dimension $a$ inclined at an angle $\alpha$ to the horizontal walls.

The upper wall of the container is located at $z=h_{T}$ and the lower wall is located at $z=-h_{B}$, where $z$ is the vertical co-ordinate. A point on the plate is the origin of a rectangular Cartesian co-ordinate system as shown in figure 1 . The elevation of the plate is

$$
z=x \tan \alpha .
$$

For convenience, let

$$
h=h_{T}+h_{B}
$$

The plate is assumed to move 'slowly' in the rotating co-ordinate frame, where the term 'slow motion' means that the Rossby number Ro $=U / \Omega a$, where $U$ is 


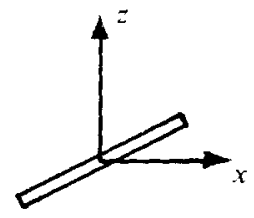

Figure 1. Geometry of the container and the plate. The upper wall is located at $z=h_{T}$ while the lower wall is the surface $z=-h_{B}$. The $y$ co-ordinate points into the page and the plate is inclined to the $x$ axis at an angle $\alpha$.

the speed characteristic of the motion of the plate, is very small. The fluid will be considered to be 'slightly' viscous or 'rapidly' rotating, which means that the Ekman number $\left(E=\nu / \Omega a^{2}\right)$ is very small. The formal limiting sequence will be to first let $R o \rightarrow 0$ and then $E \rightarrow 0$.

For small Rossby number, $\uparrow$ the equations of motion for an incompressible fluid in a rotating co-ordinate frame are

$$
\begin{gathered}
2 \boldsymbol{\Omega} \times \mathbf{u}=-\nabla p+\nu \nabla^{2} \mathbf{u}, \\
\nabla . \mathbf{u}=\mathbf{0},
\end{gathered}
$$

where $p$ is the reduced pressure defined by

$$
p=(\bar{p} / \rho)-\frac{1}{2} \Omega^{2} r^{2}
$$

The quantity $\bar{p}$ is the static pressure and $r$ is the radial distance from the origin in a horizontal plane. $\neq$ Also, $\Omega=\Omega \mathbf{k}$, where $\mathbf{k}$ is a unit vector in the vertical direction. Since (3) and (4) are linear each of the six motions corresponding to the six degrees of freedom can be studied independently and the results superposed.

The solution method will be developed by first analysing the case of horizontal translation in the $x$ direction. It is convenient to let the plate remain at rest and move the walls and the fluid at infinity with velocity

$$
\mathbf{u}_{w}=-U \mathbf{i},
$$

where $\mathbf{i}$ is a unit vector aligned with the $x$ axis.

Furthermore, to simplify the analysis, the plate will be assumed to be an ellipse inclined at the angle of attack necessary to make the Taylor column a circular cylinder. That is, if $(\xi, y, \zeta)$ are co-ordinates in a rectangular Cartesian co-ordinate system fixed on the plate defined by

$$
\begin{gathered}
\xi=x \cos \alpha+z \sin \alpha, \\
\zeta=-x \sin \alpha+z \cos \alpha,
\end{gathered}
$$

$\dagger$ If the motion is directed toward the container walls a restriction on the time scale of the motion is required in order to drop the unsteady term. This point will be discussed in $\S 3$.

¥ It is assumed in (5) that the plate is at the centre of the container. If this is not true, $r$ must be altered accordingly. Since the plate is formally regarded as having zero thickness, the calculation of forces on the plate is not affected by this term. 
then the equation of the plate is

$$
\zeta=0, \quad \xi^{2}+\left[\frac{y}{\cos \alpha}\right]^{2} \leqslant\left[\frac{a}{\cos \alpha}\right]^{2} .
$$

The projection of the region described by (9) onto the $x, y$ plane is the interior of a circle of radius $a$.

\subsection{The geostrophic flow}

In the geostrophic regions the viscous term in (3) is negligible, so that in component form (3) and (4) reduce to

$$
\begin{gathered}
-2 \Omega \bar{v}=-\partial p / \partial x \\
2 \Omega \bar{u}=-\partial p / \partial y \\
0=-\partial p / \partial z \\
\frac{\partial \bar{u}}{\partial x}+\frac{\partial \bar{v}}{\partial y}+\frac{\partial \bar{w}}{\partial z}=0
\end{gathered}
$$

where $\bar{u}, \bar{v}$ and $\bar{w}$ are the velocity components in the $x, y$ and $z$ directions, respectively. Appropriate boundary conditions are given by the Elkman compatibility condition. Hence, at the walls,

$$
\bar{w}=\left\{\begin{aligned}
-\frac{1}{2}[\nu / \Omega]^{\frac{1}{2}} \mathbf{k} \cdot \operatorname{curl}\left(\mathbf{u}-\mathbf{u}_{w}\right) & \text { on } z=h_{T}, \\
\frac{1}{2}[v / \Omega]^{\frac{1}{2}} \mathbf{k} \cdot \operatorname{curl}\left(\mathbf{u}-\mathbf{u}_{w}\right) & \text { on } z=-h_{B},
\end{aligned}\right.
$$

where $\mathbf{u}$ is the fluid velocity just outside the Ekman layer. On the upper $\left(\zeta=0^{+}\right)$ and lower $\left(\zeta=0^{-}\right)$surfaces of the plate,

$$
\bar{w}-\bar{u} \tan \alpha= \pm \frac{1}{2}\left[\frac{\nu}{\Omega \cos \alpha}\right]^{\frac{1}{2}}\left[\frac{\partial \bar{v}}{\partial x}-\frac{\partial \bar{u}}{\partial y}\right] \quad \text { on } \quad \zeta=0 \pm .
$$

Since the geostrophic equations only admit solutions for which $\mathbf{u}$ is independent of $z$ in the geostrophic region outside the Taylor column, the only way in which (14) and (15) can be satisfied simultaneously is for $\mathbf{k}$. curl $\mathbf{u}$ to vanish. Taking the divergence of the momentum equation yields (the subscript $G$ denoting a quantity in the geostrophic region outside the Taylor column)

$$
\nabla^{2} p_{G}=2 \Omega \mathbf{k} . \operatorname{curl} \mathbf{u}_{G}=0 .
$$

If cylindrical polar co-ordinates $(r, \theta, z)$ with corresponding velocity components $(u, v, w)$ are introduced, the solution for $p_{G}(r, \theta)$ is $\dagger$

$$
p_{G}(r, \theta)=2 \Omega U r \sin \theta+\sum_{n=1}^{\infty}\left[B_{n} \cos n \theta+D_{n} \sin n \theta\right]\left[\frac{a}{r}\right]^{n},
$$

where $B_{n}$ and $D_{n}$ are unknown. Once a boundary condition on the geostrophic flow on the boundary of the Taylor column has been specified, the solution for $p_{G}$ can be uniquely determined.

$\dagger$ There can be no circulation $\Gamma$ outside the Taylor column, i.e. the solution

$$
p_{G}(r, \theta) \sim 2 \Omega \Gamma \log r
$$

is not permissible. For non-zero $\Gamma$ there would be an order $E^{\frac{1}{2}}$ excess flux in the Ekman layers on the walls with no corresponding efflux (influx) to (from) the geostrophic region and mass would therefore not be conserved (Greenspan 1968). 
Letting the subscript $g$ denote quantities in the geostrophic interior of the Taylor column, the solution to (10)-(13) subject to (14)-(16) is $\dagger$

$$
\begin{gathered}
\bar{u}_{g}=O\left(E^{\frac{1}{2}}\right), \\
\bar{v}_{g}=V_{g}(x)+O\left(E^{\frac{1}{2}}\right), \\
\bar{w}_{g}=O\left(E^{\frac{1}{2}}\right), \\
p_{g}=P_{g}(x)-2 \Omega\left[\frac{\nu}{\Omega}\right]^{\frac{1}{2}} \cot \alpha\left[f^{T, B}(x) \mp \frac{1+\beta}{2} y \frac{d V_{g}(x)}{d x}\right]+O(E) \text { for } \zeta \gtreqless 0,
\end{gathered}
$$

where $\beta \equiv(\cos \alpha)^{-\frac{1}{2}}$ and

$$
d P_{g}(x) / d x=2 \Omega V_{g}(x) .
$$

The functions $V_{g}(x), f^{T^{\prime}}(x)$ and $f^{B}(x)$ are unknown functions of $x$, and the superscripts $T$ and $B$ correspond to values above and below the plate, respectively.

The fact that the pressure is continuous to leading order across vertical shear columns (Jacobs 1964) has been used in deriving (18)-(22). First, this fixes the order of magnitude of the motion inside the Taylor column. Second, because the pressure is also independent of $z$ outside the column, a discontinuity in $p_{g}$ across $\zeta=0$ is prohibited to leading order. The pressure does experience a jump of order $E^{\frac{1}{2}}$ across $\zeta=0$ as is indicated in (21). In addition to contributing to the force and moment on the plate this pressure difference will appear in the analysis of the next section, where the dynamics of the shear column will be studied.

As a third consequence of the continuity of pressure at $r=a, p_{G}(a, \theta)$ is an even function of $\theta$. This follows immediately if one notes that $p_{g}$ is independent of $y$ to order unity. However, now sufficient information is available to determine the coefficients $D_{n}$ in (17), and therefore

$$
D_{1}=-2 \Omega U a, \quad D_{n}=0 \text { for all } n \geqslant 2
$$

and (17) can be rewritten as

$$
p_{\alpha}(r, \theta)=2 \Omega U a\left[\frac{r}{a}-\frac{a}{r}\right] \sin \theta+\sum_{n=1}^{\infty} B_{n}\left[\frac{a}{r}\right]^{n} \cos n \theta .
$$

Before going on to consideration of the dynamics of the shear column (which will be necessary in order to determine $V_{g}(x), B_{n}$, etc.) it is worth while to pause and discuss the implications of the results obtained thus far. If to order one there were fluid flowing in the $x$ direction inside the Taylor column, the vortex lines would experience a change in length of order $\tan \alpha=O(1)$. However, vortex lines cannot be stretched in geostrophic regions (Greenspan 1968). Therefore, there can be no flow of order unity in the $x$ direction. This is borne out by (18). This argument places no restriction on $\bar{v}_{y}$ because no stretching occurs when vortex lines translate across the plate in the $y$ direction.

Inspection of (18)-(20) shows that the geostrophic flow inside the Taylor column is possibly rotational. For zero angle of inclination, Moore \& Saffman $(1969 b)$ found the geostrophic flow to be everywhere irrotational. This additional

$\dagger$ As an aid to providing better physical insight, all quantities will be left in dimensional form. Since all expansions (for $\alpha=O(1)$ ) will be in powers of $E$, orders of magnitude are easily traced by regarding $E\left(=\nu / \Omega a^{2}\right)$ and $\nu$ as interchangeable small parameters. 
indeterminateness manifests itself in the appearance of the unknown functions $V_{y}(x), f^{T}(x)$ and $f^{B}(x)$. The same degree of arbitrariness was found by Pedlosky \& Greenspan (1967) for a similar geometry in which closed geostrophic contours were also absent.

Finally, there is an excess flux $\mathbf{Q}$ of fluid in the Ekman layers on the plate of order $E^{\frac{1}{2}}$ given by (Greenspan 1968)

$$
\mathbf{Q} \equiv \int_{0}^{\delta E} \rho\left(\mathbf{u}-\mathbf{u}_{g}\right) d \zeta=-\frac{1}{2}\left(\frac{\nu}{\Omega \cos \alpha}\right)^{\frac{1}{2}} \rho V_{g}(x)[\mathbf{j}+\mathbf{t}],
$$

where $\delta_{E}=O\left(E_{2}^{\frac{1}{2}}\right)$ is the Ekman-layer thickness and $\mathbf{j}$ and $\mathbf{t}$ are unit vectors in the $y$ and $\xi$ directions respectively. The vector $\mathbf{Q}$ will also be referred to as the Ekman flux vector. As will be shown in $\$ 2.3$, this small flux of fluid-more specifically, the manner in which it enters the shear column-plays a central role in determining the geostrophic flow.

\subsection{The jump condition}

In order to determine the unknown functions $V_{g}(x), f^{T}(x)$ and $f^{B}(x)$ and the unknown Fourier coefficients $B_{n}$ it is necessary to examine the dynamics of the shear column. If the equations of motion are written in terms of the boundarylayer co-ordinate $\bar{x}$ centred in the shear column, i.e.

$$
\bar{x} \equiv r-a,
$$

then since the shear column appears to be locally plane the approximate equations of motion become

$$
\begin{gathered}
-2 \Omega v=-\frac{\partial p}{\partial \bar{x}}+\nu \frac{\partial^{2} u}{\partial \bar{x}^{2}} \\
2 \Omega u=-\frac{1}{a} \frac{\partial p}{\partial \theta}+\nu \frac{\partial^{2} v}{\partial \bar{x}^{2}} \\
O=-\frac{\partial p}{\partial z}+\nu \frac{\partial^{2} w}{\partial \bar{x}^{2}} \\
\frac{\partial u}{\partial \bar{x}}+\frac{1}{a} \frac{\partial v}{\partial \theta}+\frac{\partial w}{\partial z}=0
\end{gathered}
$$

where $u, v$ and $w$ are the velocity components in the $r, \theta$ and $z$ directions, respectively.

The shear column is a ' $\frac{1}{3}$-layer', $\dagger$ which has thickness of order $E^{\frac{1}{3}}$. Hence, the viscous term in (26) is negligible, whereby the radial momentum balance simplifies to

$$
-2 \Omega v=-\partial p / \partial \bar{x} \text {. }
$$

Equations (27)-(30) can be combined to yield the shear-column equations

$$
\begin{aligned}
& \frac{\partial w}{\partial z}=-\frac{\nu}{2 \Omega} \frac{\partial^{3} v}{\partial \bar{x}^{3}}, \\
& \frac{\partial v}{\partial z}=\frac{\nu}{2 \Omega} \frac{\partial^{3} w}{\partial \bar{x}^{3}} .
\end{aligned}
$$

$\dagger$ If the additional hypothesis that $E^{\frac{1}{12}} \ll 1$ is made, the $\frac{1}{3}$-layer is enveloped by a ' $\frac{1}{4}$-layer' (thickness $\sim E \frac{E}{\text { ) }}$ ) on the outer edge of the shear column. This physically uninteresting constraint will not be placed on the Ekman number in this study. 
The Ekman condition is valid in the $\frac{1}{3}$-layer (Stewartson 1966) and in boundarylayer co-ordinates, (14)-(16) simplify to

$$
w=\left\{\begin{array}{l}
-\frac{1}{2}[v / \Omega]^{\frac{1}{2}} \partial v / \partial \bar{x} \text { on } z=h_{T}, \\
-(v \sin \theta-u \cos \theta) \tan \alpha \pm \frac{1}{2}[v / \Omega \cos \alpha]^{\frac{1}{2}} \partial v / \partial \bar{x} \quad \text { on } \zeta=0^{ \pm}, \bar{x}<0, \\
\frac{1}{2}[v / \Omega]^{\frac{1}{2}} \partial v / \partial \bar{x} \text { on } z=-h_{B} .
\end{array}\right.
$$

If perturbation expansions for $v$ and $w$ (presumably in powers of $E^{\frac{1}{6}}$ ) are introduced a hierarchy of Wiener-Hopf problems results. This is more difficult than the hierarchy studied by Moore \& Saffman (1969a) because of the inhomogeneous boundary condition at the plate. Rather than attempting to solve for the detailed structure of the shear column, the techniques developed by Stewartson (1966) can be used. His basic idea is to integrate the shear-column equations across the $\frac{1}{3}$-layer to determine jump conditions relating the solutions inside and outside the Taylor column. The physical significance of the various jump conditions has been elucidated by Moore \& Saffman (1969a).

In the present application a single jump condition involving conservation of mass in the shear column is sufficient to determine the geostrophic flow. It can be derived by considering the excess flux of fluid from the Ekman layers on the plate. The question of where the excess flux goes is of particular concern.

In their studies, Moore \& Saffman considered the possibility of the fluid swirling around the edge of the plate in the region where the $\frac{1}{3}$-layer and the Ekman layer merge. This region is referred to as a 'collar'. Conservation of mass would require a velocity of order $E^{-\frac{1}{2}}$, which entails viscous dissipation of order unity. However, there is no mechanism to balance this dissipation, so that the possibility of a collar is ruled out. The possibility of a radial jet can be rejected on the same grounds.

Hence Moore \& Saffman argued that, to escape from the Ekman layers on the plate, the fluid erupts as a vertical jet into the $\frac{1}{3}$-layer. This structure is assumed to be valid for the problem of interest here.

To facilitate understanding of how these ideas are applied in the present context a control volume about the edge of the plate is shown in figure 2. The control volume encloses the Ekman layers on the upper and lower surfaces of the plate and the lateral boundaries are the edges of the $\frac{1}{3}$-layer. All relevant fluxes of fluid across the control-volume boundaries are also shown. Viscous dissipation in the $\frac{1}{3}$-layer should be $o(1)$ so that, necessarily, $v=o\left(E^{-\frac{1}{3}}\right)$. Hence, the circumferential flux through the control volume need not be considered as it is $o\left(E^{\frac{1}{2}}\right)$.

The amount of fluid entering the control volume from the Ekman layers on the plate is

$$
\int_{-\delta E}^{\delta_{E} E} \rho \mathbf{u} \cdot \mathbf{n}_{e} \frac{d \zeta}{\cos \alpha}=\int_{-\delta_{E}}^{\delta_{E}} \rho \mathbf{u}_{g} \cdot \mathbf{n}_{e} \frac{d \zeta}{\cos \alpha}+Q_{e}(\theta)
$$

where $Q_{\ell}(\theta)$ is the excess flux and $\mathbf{n}_{e}$ is a unit vector normal to the Taylor column. For the special geometry considered here, $\mathbf{n}_{e}$ is in the radial direction. The excess flux is given by

$$
Q_{e}(\theta)=2 \mathbf{n}_{e} \cdot \mathbf{Q} / \cos \alpha
$$

where $\mathbf{Q}$ is the Ekman flux vector defined in (24). 


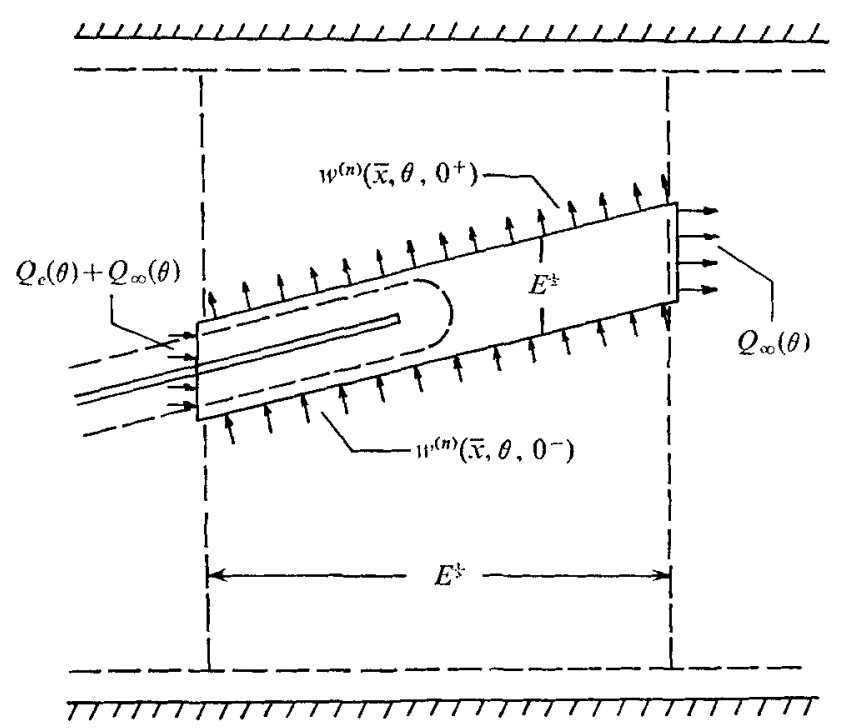

FIgure 2. Control volume in the $\frac{1}{3}$-layer and the relevant fluxes of fluid across its boundaries. $Q_{e}(\theta)$ is the excess flux from the Ekman layers on the plate, $Q_{\infty}(\theta)$ is the flux in the absence of Ekman layers and $w^{(n)}(\bar{x}, \theta, \zeta)$ is the velocity component normal to the plate.

The amount of fluid passing across the vertical boundary of the control volume at the outer edge of the shear column is

$$
\int_{-\delta_{E}}^{\delta E} \rho \mathbf{u}_{G} \cdot \mathbf{n}_{e} \frac{d \zeta}{\cos \alpha}
$$

In general, the velocity component normal to a detached vertical shear column is continuous across the column to leading order (Jacobs 1964) and therefore

$$
\int_{-\delta E}^{\delta_{E}} \rho \mathbf{u}_{g} \cdot \mathbf{u}_{e} \frac{d \zeta}{\cos \alpha}=\int_{-\delta E}^{\delta_{E}} \rho \mathbf{u}_{G} \cdot \mathbf{n}_{e} \frac{d \zeta}{\cos \alpha} \equiv Q_{\infty}(\theta)
$$

In order for mass to be conserved in the $\frac{1}{3}$-layer, the excess flux $Q_{e}(\theta)$ must therefore be balanced by the net flux of fluid across the boundaries of the control volume which are parallel to the plate. In terms of the velocity component $w^{(n)}(\bar{x}, \theta, \zeta)$ normal to the plate defined by

$$
w^{(n)} \sec \alpha=w+(v \sin \theta-u \cos \theta) \tan \alpha,
$$

the most fundamental form of the jump condition which is needed to relate the geostrophic regions on the two sides of the shear column is

$$
Q_{e}(\theta)=\int_{-\infty}^{\infty} \rho\left[w^{(n)}\left(\bar{x}, \theta, 0^{+}\right)-w^{(n)}\left(\bar{x}, \theta, 0^{-}\right)\right] \sec \alpha d \bar{x},
$$

where $\bar{x}= \pm \infty$ corresponds to the edges of the $\frac{1}{3}$-layer in the usual matched asymptotic expansion sense. Equation (39) can be applied to any geometry provided only that the unit vector $\mathbf{n}_{e}$ used in evaluating $Q_{e}(\theta)$ is the local unit normal to the Taylor column.

The techniques developed by Stewartson (1966) for analysing detached vertical shear columns can be used to express the right-hand side of (39) in terms of the 
geostrophic flow variables. This is accomplished in the appendix, and (39) reduces to the more useful form

$$
\frac{Q_{e}(\theta)}{\rho}=\left[\frac{\nu}{\Omega}\right]^{\frac{1}{2}}\left[v_{g}(a, \theta)-v_{G}(a, \theta)\right]-\tan \alpha \sin \theta \frac{\Delta p_{g}(a, \theta)}{2 \Omega},
$$

where $v_{g}(r, \theta)$ and $v_{G}(r, \theta)$ are the azimuthal velocity components of $\mathbf{u}_{g}$ and $\mathbf{u}_{G}$ respectively and $\Delta p_{g}(a, \theta)$ is the jump in $p_{g}$ across $\zeta=0$ at the edge of the plate. Equation (40) is also applicable to any geometry provided that $v$ is the velocity component in the direction tangent to the Taylor column and $\theta$ is the angle between $\mathbf{n}_{e}$ and the $x$ axis.

Since the Ekman flux $Q_{e}(\theta)$ is a function only of the geostrophic flow inside the Taylor column (through its dependence upon $\mathbf{Q}$ ) and the geometry of the plate (through its dependence upon $\mathbf{n}_{e}$ ), all the terms appearing in (40) can be expressed in terms of the geostrophic variables. This is the desired jump condition relating the geostrophic regions.

To evaluate $Q_{e}(\theta)$, note that by definition

$$
\begin{gathered}
u_{g}(a, \theta)=V_{g}(a \cos \theta) \sin \theta=u_{G}(a, \theta), \\
v_{g}(a, \theta)=V_{g}(a \cos \theta) \cos \theta=u_{G}(a, \theta) \cot \theta .
\end{gathered}
$$

The fact that $u$ is continuous across the shear column to first order has been used in writing (41). In terms of $u_{G}(a, \theta)$, the excess flux becomes

where $\beta \equiv(\cos \alpha)^{-\frac{1}{2}}$.

$$
Q_{e}(\theta)=-\beta \rho(v / \Omega)^{\frac{1}{2}}\left[\beta^{2}+\cot \theta\right] u_{G}(a, \theta),
$$

The quantity $\Delta p_{g}(a, \theta)$ can be evaluated from (21), and the result is

$\Delta p_{g}(a, \theta)=-2 \Omega\left(\frac{\nu}{\Omega}\right)^{\frac{1}{2}} \cot \alpha\left\{f^{T}(a \cos \theta)-f^{B}(a \cos \theta)+(1+\beta) \frac{d}{d \theta}\left[u_{G}(a, \theta) \operatorname{cosec} \theta\right]\right\}$.

Substitution of (42)-(44) into (40) yields the more simplified version of the jump condition:

$$
(1+\beta) \frac{d u_{G}(a, \theta)}{d \theta}+\beta^{3} u_{G}(a, \theta)-v_{G}(a, \theta)=-\left[f^{T}(a \cos \theta)-f^{B}(a \cos \theta)\right] \sin \theta .
$$

The additional information contained in (45) will be shown in $\$ 2.4$ to be sufficient to remove the indeterminateness of the geostrophic solution.

\subsection{The solution}

The velocity components $u_{G}(r, \theta)$ and $v_{G}(r, \theta)$ follow from substitution of $p_{G}(r, \theta)$ into the geostrophic equations:

$$
\begin{gathered}
u_{G}(a, \theta)=\frac{1}{2 \Omega a} \sum_{n=1}^{\infty} n B_{n} \sin n \theta \\
v_{G}(a, \theta)=2 U \sin \theta-\frac{1}{2 \Omega a} \sum_{n=1}^{\infty} n B_{n} \cos n \theta .
\end{gathered}
$$

Inspection of (45)-(47) reveals an interesting fact. Since $f^{T}(a \cos \theta)$ and $f^{B}(a \cos \theta)$ 
are by definition even functions of $\theta$, the right-hand side of (45) is necessarily odd in $\theta$. However, the left-hand side of (45) involves both terms which are even in $\theta$ and terms which are odd in $\theta$. Hence, the sum of the even terms must vanish while the odd terms sum to the right-hand side of (45). That is, (45) yields the following two equations:

$$
\begin{gathered}
(1+\beta) \frac{d u_{G}(a, \theta)}{d \theta}=v_{G}(a, \theta)-2 U \sin \theta \\
f^{T}(a \cos \theta)-f^{B}(a \cos \theta)=2 U-\beta^{3} u_{G}(a, \theta) \operatorname{cosec} \theta .
\end{gathered}
$$

Substitution of (46) and (47) into (48) yields

from which

$$
\sum_{n=1}^{\infty} n[(1+\beta) n+1] B_{n} \cos n \theta=0
$$

Therefore, $u_{G}(a, \theta)$ vanishes and, from (41), $V_{g}(a \cos \theta)$ is also zero, i.e.

$$
V_{g}(x)=0 \text {. }
$$

This shows that to leading order the fluid inside the Taylor column is motionless relative to the plate. The flow outside the Taylor column is the potential flow of a uniform stream past a circular cylinder given by

$$
\begin{gathered}
u_{G}(r, \theta)=-U\left[1-(a / r)^{2}\right] \cos \theta \\
v_{G}(r, \theta)=U\left[1+(a / r)^{2}\right] \sin \theta
\end{gathered}
$$

The force and moment on the disk can be calculated by noting from (49) that

$$
f^{T}(x)-f^{B}(x)=2 U,
$$

so that the pressure difference between the top and bottom of the disk is known in addition to the viscous stress difference. The force (for an $x$ translation) is

while the moment is

$$
\mathbf{F}_{x}=4 \pi \rho \Omega a^{2} U(\nu / \Omega)^{\frac{1}{2}}[-\mathbf{i}+\mathbf{k} \cot \alpha]
$$

$$
\mathbf{M}_{x}=o\left(E^{\frac{1}{2}}\right) \text {. }
$$

It is possible to solve for general slow motion of the elliptical plate by using the same technique. However, the results are of limited value because of the relationship between the angle of attack and the shape of the plate. This relationship is of no importance for infinitesimal $\alpha$, where the plate can be replaced by a disk with the Taylor column remaining circular to a first approximation. In $\$ 3$ the fluid motion resulting when a disk moves using all six degrees of freedom at infinitesimal angle of attack is discussed.

Before going on to a discussion of the limiting case of small $\alpha$, the jump condition will be cast in a more general form.

\subsection{Generalization of the jump condition}

Although the jump condition has been derived for a very special geometry, it can be applied to much more general situations. As was noted in $\$ 2.3$, none of the special features of the cylindrical symmetry of the Taylor column have been used 
in arriving at the jump condition as expressed in (40). It can be used to calculate the fluid motion ensuing from general slow motion of a thin plate having any shape at an arbitrary angle of attack with the following generalized definitions.

(i) The velocity vector is written as $\mathbf{u}=(u, v, w)$ at the edges of the shear column, where $u$ and $v$ are the horizontal components of $\mathbf{u}$ normal to and tangent to the Taylor column respectively and $w$ is the component of $\mathbf{u}$ in the vertical direction.

(ii) $\mathbf{n}_{e}$ is a unit vector in a horizontal plane normal to the Taylor column.

(iii) $\theta$ is the local angle between $\mathbf{n}_{e}$ and the $x$ axis and $a$ is the local radius of curvature of the Taylor column.

(iv) The Ekman flux vector $\mathbf{Q}$, which is needed to calculate $Q_{e}(\theta)$, is evaluated from the general expression (Greenspan 1968)

$$
\mathbf{Q}=\frac{\mathbf{1}}{2}\left[\nu / \mid \boldsymbol{\Omega} \cdot \mathbf{n}[]^{\frac{1}{2}} \mathbf{n} \times\left[\left(\mathbf{u}-\mathbf{u}_{p}\right)+\mathbf{n} \times\left(\mathbf{u}-\mathbf{u}_{p}\right)\right],\right.
$$

where $\mathbf{u}_{p}$ is the plate velocity and $\mathbf{n}$ is the unit normal to the plate:

$$
\mathbf{n}=-\mathbf{i} \sin \alpha+\mathbf{k} \cos \alpha \text {. }
$$

\section{Motion of a disk at an infinitesimal angle of attack}

A brief account of the details of general slow motion of a disk when $\alpha$ is small is given in this section.

\subsection{Simplification of the jump condition}

For a very small angle of attack, considerable simplification of the shear-column analysis is possible and the jump condition can be derived more rigorously (Wilcox 1970). A lower bound must be placed on $\alpha$ to ensure that the geostrophic flow has the same structure as in $\S 2$. That is, $\alpha$ must be very large compared with the Ekman-layer thickness to guarantee the absence of closed geostrophic contours between the disk and the walls. Hence, $\alpha$ must be restricted to the range

$$
E^{\frac{1}{2}} \ll \alpha \ll 1 \text {. }
$$

A modest simplification of the Ekman flux vector $\mathbf{Q}$ given by (58) is possible. When the disk does not move toward the horizontal walls (horizontal translation and rotation about the vertical axis), it is convenient to work in disk-fixed coordinates. Hence, $\mathbf{u}_{p}$ vanishes. For the other two rotations and for vertical translation it is more convenient to work in container-fixed co-ordinates. In these three cases the geostrophic flow has been shown to be of order $\cot \alpha$ (Wilcox 1970). Since $u_{p}$ is of $O(1)$, it can be neglected for these three motions. Therefore, the excess flux from the Ekman layers on the disk which enters the shear column is

$$
Q_{e}(\theta) \doteq-\rho(\nu / \Omega)^{\frac{1}{2}}\left[u_{G}(a, \theta)+v_{g}(a, \theta)\right]
$$

Combining (40) and $(60)$, the jump condition reduces to

$$
2 v_{g}(a, \theta)+u_{G}(a, \theta)-v_{G}(a, \theta)=(\Omega / \nu)^{\frac{1}{2}} \tan \alpha \sin \theta\left[\Delta p_{g}(a, \theta) / 2 \Omega\right] .
$$




\subsection{Horizontal translation}

For translation in the $x$ direction the geostrophic solution given in $\S 2$ is valid $\dagger$ with $\beta \rightarrow 1$ for small $\alpha$. Hence, the jump condition reduces to

$$
2 d u_{G}(a, \theta) / d \theta+u_{G}(a, \theta)-v_{G}(a, \theta)=-\left[f^{T}(a \cos \theta)-f^{B}(a \cos \theta)\right] \sin \theta
$$

and a solution exists only if the terms on the left-hand side of $(62)$ which are even in $\theta$ sum to zero. Therefore,

$$
\sum_{n=1}^{\infty} n(2 n+1) B_{n} \cos n \theta=0
$$

which is the limiting form of (50) for $\alpha \rightarrow 0$, and the unique solution is again $B_{n}=0$ for all $n \geqslant 1$.

The force on the disk is given by equation (56) and the moment is

$$
\mathbf{M}_{x}=o\left(E^{\frac{1}{2}} \cot \alpha\right) \text {. }
$$

For translation in the $y$ direction it is convenient to work in disk-fixed coordinates. Hence, the disk remains at rest and the walls and fluid at infinity move with velocity

$$
\mathbf{u}_{v}=-V \mathbf{j} \text {. }
$$

The geostrophic solution for $r<a$ is again given by (18)-(22) and the jump condition by (62). The geostrophic flow outside the Taylor column is different and (46) and (47) must be replaced by

$$
\begin{aligned}
& u_{G}(a, \theta)=-V \sin \theta+\frac{1}{2 \Omega a} \sum_{n=1}^{\infty} n B_{n} \sin n \theta, \\
& v_{G}(a, \theta)=-V \cos \theta-\frac{1}{2 \Omega a} \sum_{n=1}^{\infty} n B_{n} \cos n \theta .
\end{aligned}
$$

Proceeding in the now established manner, it follows that

$$
\frac{1}{2 \Omega a} \sum_{n=1}^{\infty} n(2 n+1) B_{n} \cos n \theta=V \cos \theta
$$

therefore

$$
B_{1}=\frac{2}{3} \Omega a V, \quad B_{n}=0 \text { for all } n \geqslant 2 .
$$

Hence, the geostrophic flow inside the Taylor column is given by

$$
\bar{v}_{g}=-\frac{2}{3} V, \quad \bar{u}_{g}=\bar{w}_{g}=o\left(E^{\frac{1}{2}} \cot \alpha\right) .
$$

This shows that fluid passes through the column and experiences a deceleration to two-thirds of the free-stream velocity. The geostrophic flow for $r>a$ is

$$
\begin{aligned}
& u_{G}(r, \theta)=-V\left[1-\frac{1}{3}(a / r)^{2}\right] \sin \theta, \\
& v_{G}(r, \theta)=-V\left[1+\frac{1}{3}(a / r)^{2}\right] \cos \theta
\end{aligned}
$$

and the streamlines are as shown in figure 3.

An interesting feature of this motion is the special form of the force on the disk, i.e.

$$
\mathbf{F}_{y}=\frac{4}{3} \pi \rho \Omega \alpha^{2} V(v / \Omega)^{\frac{1}{2}} \cot \alpha \mathbf{k},
$$

which means that, to leading order $\left[O\left(E^{\frac{1}{2}}\right)\right]$, there is no drag. As in the case of $x$ translation, the moment vanishes to order $E^{\frac{1}{2}} \cot \alpha$.

$\dagger$ This statement is true with the qualification that, for small $\alpha$, the perturbation expansions in the geostrophic regions proceed in powers of $E^{\frac{1}{2}} \cot \alpha$ rather than $E^{\frac{1}{2}}$. 


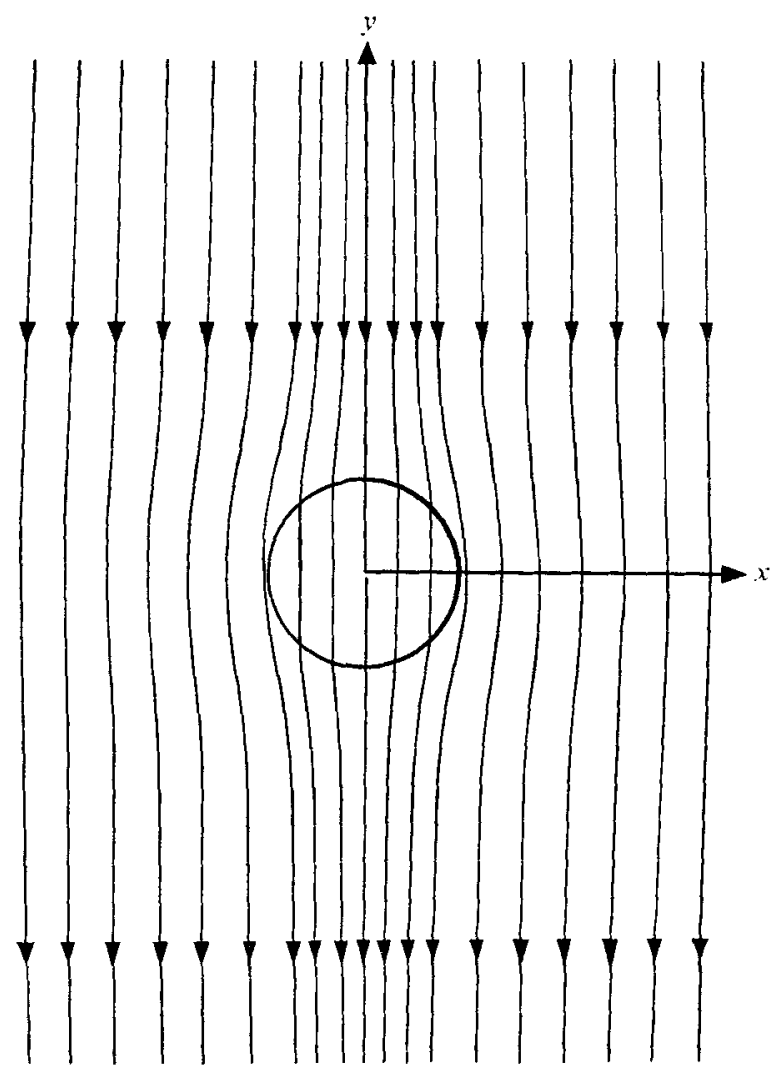

Figure 3. Geostrophic flow for $y$ translation (disk at rest).

\subsection{Vertical translation}

All relevant notation used in $\$ 2$ will again be used. The disk will be assumed to move vertically with speed $W$ so that

$$
\mathbf{u}_{p}=W \mathbf{k} \text {. }
$$

The walls and fluid at infinity remain at rest.

Since vortex lines cannot be stretched in the geostrophic regions between the walls and the disk, necessarily

$$
\bar{u}_{g}=-W \cot \alpha \text {. }
$$

This permits vortex lines to preserve their length by translating across the disk in the $x$ direction as its elevation changes. $\dagger$

This motion is inherently unsteady as the geometry is changing with time. However, if the time scale of the motion, $\tau \sim a / W \cot \alpha$, is assumed to satisfy $\tau \gg 1 / \Omega$ or, equivalently,

$$
R_{0} \ll \tan \alpha,
$$

$\uparrow$ This result is consistent with the analysis of Moore \& Saffman (1969a) for a disk rising with zero angle of attack. They found that the horizontal fluid velocity inside the Taylor column is of order $E^{-\frac{1}{2}}$, indicating a smooth transition through the regime where $\alpha \sim E^{\frac{1}{2}}$. 


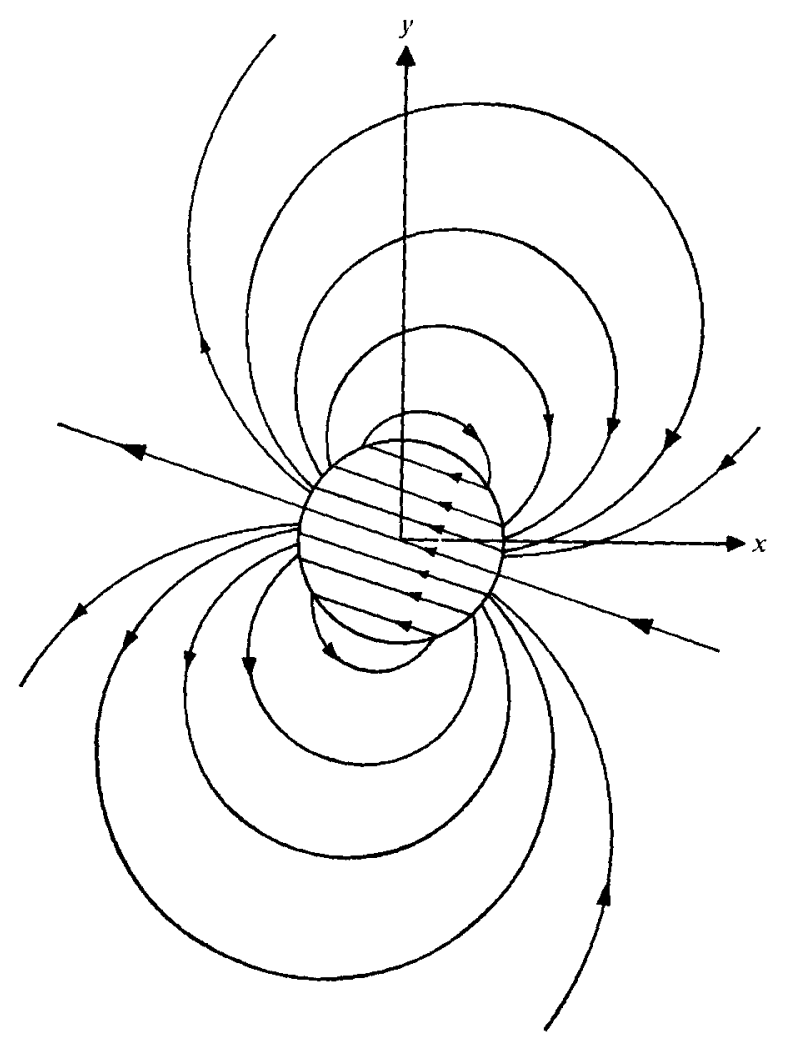

FigURE 4. Geostrophic flow for vertical translation (disk moving).

the unsteady term (in addition to the nonlinear inertia term) in the momentum equation can be neglected. Furthermore, the distance from the disk to the walls is unchanged to a first approximation, i.e. the solution obtained will be a quasisteady solution.

As with horizontal translation, the geostrophic equations and the Ekman compatability relation do not yield sufficient information to determine the geostrophic solution. Expanding in Fourier series and using the jump condition, the solution evolves as

$$
\bar{v}_{g}=\frac{1}{3} W \cot \alpha, \quad \bar{w}_{y}=O\left(E^{\frac{1}{2}} \cot \alpha\right)
$$

Thus, the geostrophic flow is inclined to the $x$ axis at an angle

$$
-\tan ^{-1} \frac{1}{3} \doteq-18 \cdot 4^{\circ} \text {. }
$$

The geostrophic flow outside the column is

$$
\begin{aligned}
& u_{G}(r, \theta)=-W \cot \alpha\left[\cos \theta-\frac{1}{3} \sin \theta\right](a / r)^{2}, \\
& v_{G}(r, \theta)=-W \cot \alpha\left[\sin \theta+\frac{1}{3} \cos \theta\right](a / r)^{2}
\end{aligned}
$$

and the force and moment on the disk are

$$
\begin{gathered}
\mathbf{F}_{z}=-\frac{2}{3} \pi \rho \Omega a^{2} W(\nu / \Omega)^{\frac{1}{2}} \cot ^{2} \alpha[-9 \tan \alpha \mathbf{i}+3 \tan \alpha \mathbf{j}+10 \mathbf{k}], \\
\mathbf{M}_{z}=o\left(E^{\frac{1}{2}} \cot ^{2} \alpha\right) .
\end{gathered}
$$

The streamlines are as shown in figure 4. 


\subsection{The three rotations}

The term ' $x$ rotation' will denote the motion corresponding to

$$
\mathbf{u}_{p}=\epsilon_{x} \Omega \mathbf{i} \times \mathbf{r}
$$

while $y$ and $z$ rotation denote the motions corresponding to

and

$$
\begin{aligned}
& \mathbf{u}_{p}=\epsilon_{y} \Omega \mathbf{j} \times \mathbf{r} \\
& \mathbf{u}_{p}=\epsilon_{z} \Omega \mathbf{k} \times \mathbf{r}
\end{aligned}
$$

respectively. To justify neglect of the unsteady and nonlinear inertia terms in the momentum equation, necessarily $\epsilon_{x}, \epsilon_{y} \ll \tan \alpha$ and $\epsilon_{z} \ll 1$. In the same quasisteady sense as in the vertical translation analysis, the geometrical configuration (including angle of attack) is unchanged.

The solutions for the three rotations are given below in terms of the reduced pressure (which is proportional to the stream function in geostrophic regions). The flow is of order cot $\alpha$ for $x$ and $y$ rotation while it is of order unity for $z$ rotation.

$$
p_{g}(x, y)=\left\{\begin{array}{ll}
\epsilon_{x} \Omega^{2} a^{2}\left[(y / a)^{2}+\frac{1}{5}(x / a)^{2}-\frac{3}{5}\right] \cot \alpha, & x \text { rotation, } \\
2 \epsilon_{y} \Omega^{2} a^{2}\left[\frac{1}{5}(x / a)^{2}-(x / a)(y / a)-\frac{1}{10}\right] \cot \alpha, & y \text { rotation, } \\
-\frac{1}{5} \epsilon_{z} \Omega^{2} a^{2}\left[7(x / a)^{2}+5(y / a)^{2}-6\right], & z \text { rotation, }
\end{array}\right\}
$$

inside the Taylor column, and

$$
p_{G}(r, \theta)=\left\{\begin{array}{ll}
-\frac{2}{5} \epsilon_{x} \Omega^{2} a^{2} \cot \alpha(a / r)^{2} \cos 2 \theta, & x \text { rotation, } \\
-\epsilon_{y} \Omega^{2} a^{2} \cot \alpha(a / r)^{2}\left[\sin 2 \theta-\frac{1}{5} \cos 2 \theta\right], & y \text { rotation, } \\
-\frac{1}{5} \epsilon_{z} \Omega^{2} a^{2}(a / r)^{2} \cos 2 \theta, & z \text { rotation, }
\end{array}\right\}
$$

for $r>a$. The streamlines are sketched in figures 5,6 and 7 . The moments on the disk are

$$
\mathbf{M}=\left\{\begin{array}{ll}
-\frac{2}{5} \pi \rho \epsilon_{x} \Omega^{2} a^{4}(\nu / \Omega)^{\frac{1}{2}} \cot ^{2} \alpha[3 \mathbf{i}+\mathbf{j}+3 \tan \alpha \mathbf{k}], & x \text { rotation, } \\
-\frac{1}{10} \pi \rho \epsilon_{y} \Omega^{2} a^{4}(\nu / \Omega)^{\frac{1}{2}} \cot ^{2} \alpha[4 \mathbf{i}+28 \mathbf{j}-\tan \alpha \mathbf{k}], & y \text { rotation, } \\
\frac{1}{5} \pi \rho \epsilon_{z} \Omega^{2} a^{4}(\nu / \Omega)^{\frac{1}{2}} \cot \alpha[7 \mathbf{i}-\mathbf{j}+7 \tan \alpha \mathbf{k}], & z \text { rotation, }
\end{array}\right\}
$$

and in all cases the force on the disk vanishes to leading order $\left[O\left(E^{\frac{1}{2}} \cot ^{2} \alpha\right)\right.$ for $x$ and $y$ rotation and $O\left(E^{\frac{1}{2}} \cot \alpha\right)$ for $z$ rotation].

\subsection{Motion when the upper surface is free}

It is appropriate to mention here the results when the upper surface is free. If the surface tension is large enough to keep the surface plane to within $O\left(E^{\frac{1}{2}}\right)$, the free surface cannot sustain an Ekman layer. This is true because the geostrophic flow satisfies the condition that the tangential stress vanishes on a free surface. Hence, (14) must be replaced by

$$
w=0 \quad \text { on } \quad z=h_{T} .
$$

In the case of horizontal translation, for example, the form of the geostrophic solution is unaffected to order one, so that (18)-(22) still apply. However, the limiting form of (44) must be replaced by

$\Delta p_{g}(a, \theta)=-2 \Omega\left(\frac{\nu}{\Omega}\right)^{\frac{1}{2}} \cot \alpha\left\{f^{T}(a \cos \theta)-f^{B}(a \cos \theta)+\frac{3}{2} \frac{d}{d \theta}\left[u_{G}(a, \theta) \operatorname{cosec} \theta\right]\right\}$ 


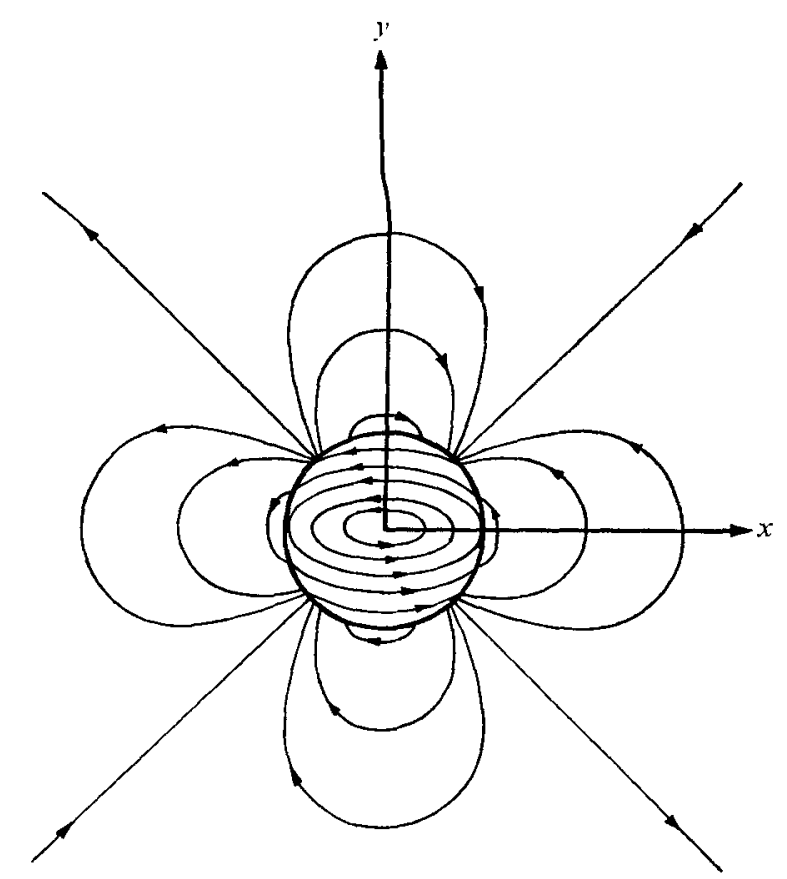

Figure 5. Geostrophic flow for $x$ rotation (disk moving).

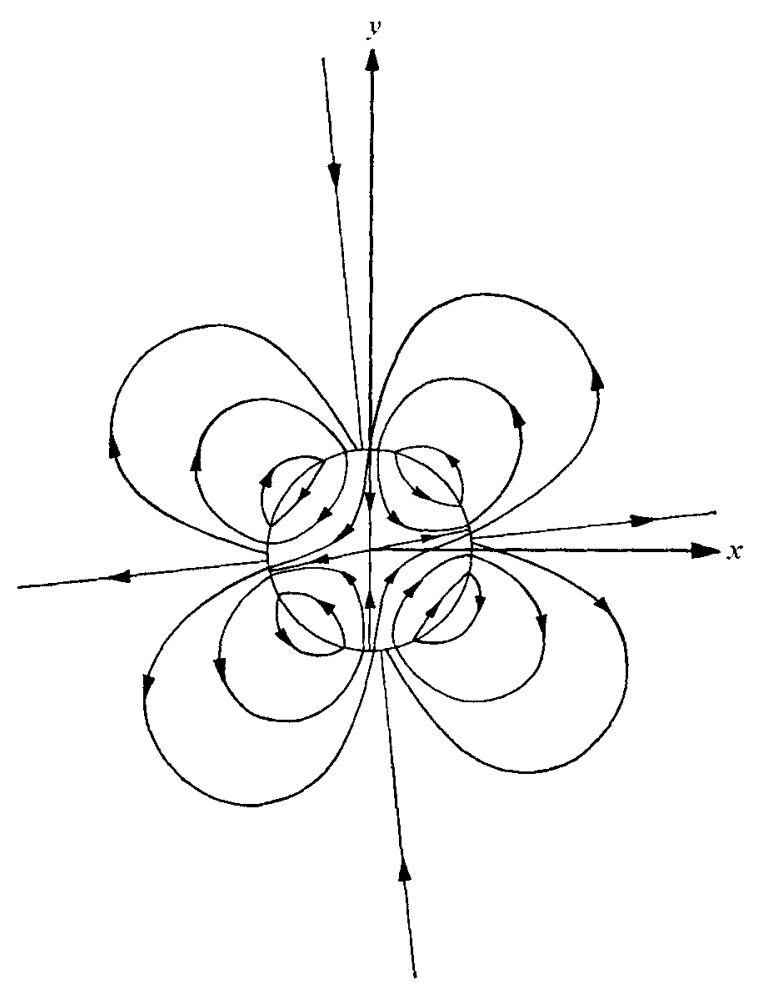

FIGURE 6. Geostrophic flow for $y$ rotation (disk moving). 


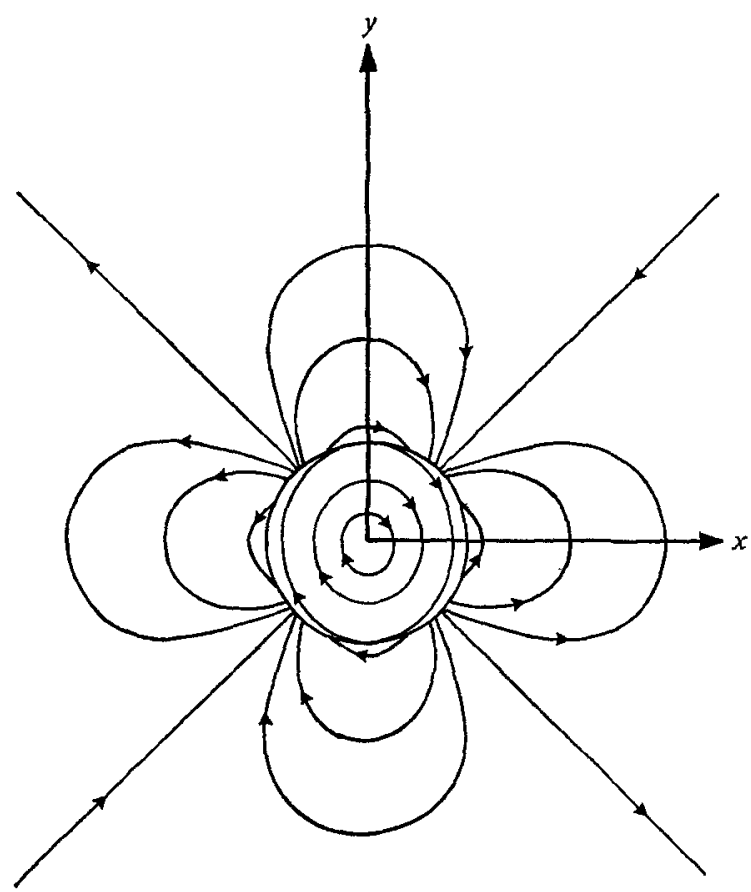

Figure 7. Geostrophic flow for $z$ rotation (disk moving).

and (61) must be modified to

$$
\frac{3}{2} v_{g}(a, \theta)+u_{G}(a, \theta)-v_{G}(a, \theta)=(\Omega / \nu)^{\frac{1}{2}} \tan \alpha \sin \theta\left[\Delta p_{g}(a, \theta) / 2 \Omega\right],
$$

so that the equation corresponding to $(62)$ is

$$
\frac{3}{2} d u_{G}(a, \theta) / d \theta+u_{G}(a, \theta)-v_{G}(a, \theta)=-\left[f^{T}(a \cos \theta)-f^{B}(a \cos \theta)\right] \sin \theta .
$$

For $x$ translation the geostrophic flow is unchanged. For $y$ translation the geostrophic flow inside the Taylor column is given by

$$
\bar{v}_{g}=-\frac{4}{5} V, \quad \bar{u}_{g}=\bar{w}_{g}=O\left(E^{\frac{1}{2}} \cot \alpha\right)
$$

and the force on the disk becomes

$$
\mathbf{F}=\frac{8}{5} \pi \rho \Omega a^{2} V(\nu / \Omega)^{\frac{1}{2}} \cot \alpha \mathbf{k},
$$

so that there is no drag to leading order on the disk just as with the rigid upper surface.

In the case of $z$ translation and the three rotations the qualitative features of the solution remain the same with the exception that the flow inside the Taylor column for vertical translation is inclined to the $x$ axis at an angle

$$
-\tan ^{-1} \frac{2}{5} \doteq-21 \cdot 8^{\circ} \text {. }
$$




\section{Discussion}

The most important result obtained in this study is the technique developed in $\$ 2$ for analysing slow motion of a plate at a finite angle of attack $\alpha$. In particular, the jump condition [equation (40)] obviates the necessity of examining the detailed structure of the shear column separating the geostrophic regions inside and outside the Taylor column. With appropriately generalized definitions as discussed in $\$ 2.5$, equation (40) can be used to analyse general slow motion of any thin plate at an arbitrary angle of attack. The solution method is especially simple for a circular Taylor column.

For an elliptical plate inclined at the angle of attack required to generate a circular Taylor column, horizontal translation in the $x$ direction (see figure 1) has been shown to render a stagnant Taylor column. This result is valid for an infinitesimal angle of attack (provided $\alpha \gg E^{\frac{1}{2}}$ ), in which case the limiting form of the elliptical plate is a disk.

The salient features of disk motion at a small angle of attack using the other five degrees of freedom have been given in $\S 3$. Some of the most important points are the following.

(i) For motion other than $x$ translation there is flow through the Taylor column.

(ii) When the distance between the disk and the horizontal walls changes ( $z$ translation, $x$ and $y$ rotation), the horizontal geostrophic velocity is of order $\cot \alpha$; otherwise it is of order unity.

(iii) When the disk translates in the $y$ direction there is no drag.

The author is greatly indebted to Professor P. G. Saffman of the California Institute of Technology for his patient assistance and his helpful comments and suggestions during the course of this work.

\section{Appendix}

The purpose of this appendix is to evaluate the integral appearing in (39) in terms of the geostrophic flow variables. For any quantity $\phi(\bar{x}, \theta, \zeta)$ the jump in $\phi$ across $\zeta=0$ will be denoted by $\Delta \phi$, i.e.

$$
\Delta \phi \equiv \phi\left(\bar{x}, \theta, 0^{+}\right)-\phi\left(\bar{x}, \theta, 0^{-}\right) .
$$

Also, to further simplify the notation, $\phi\left(\bar{x}, \theta, \zeta=0^{ \pm}\right), \phi\left(\bar{x}, \theta, z=h_{T}\right)$ and

$$
\phi\left(\bar{x}, \theta, z=-h_{B}\right)
$$

will be denoted as $\phi\left(0^{ \pm}\right), \phi\left(h_{T}\right)$ and $\phi\left(-h_{B}\right)$, respectively.

Since the velocity component normal to the Taylor column is continuous across the column to leading order, necessarily

$$
u=u_{G}(a, \theta)+O\left(E^{\frac{1}{3}}\right) .
$$

Therefore, $\Delta u=O\left(E^{\frac{1}{3}}\right)$ and from (38) it follows that

$$
\Delta w^{(n)} \sec \alpha=\Delta w+\Delta v \sin \theta \tan \alpha+O\left(E^{\frac{1}{3}}\right) .
$$


Equation (30) can be used to express $\Delta v$ as

$$
\Delta v=(1 / 2 \Omega) \partial(\Delta p) / \partial \bar{x} .
$$

An expression for $\Delta w$ can be derived by integrating (31) in the $z$ direction in the following manner:

$$
\int_{z=-h_{B}}^{\zeta=0^{-}} \frac{\partial w}{\partial z} d z=w\left(0^{-}\right)-w\left(-h_{B}\right)=-\frac{\nu}{2 \Omega} \frac{\partial}{\partial \bar{x}} \int_{z=-h_{B}}^{\zeta=0^{-}} \frac{\partial^{2} v}{\partial \bar{x}^{2}} d z
$$

and

$$
\int_{\zeta=0^{+}}^{z=h T} \frac{\partial w}{\partial z} d z=w\left(h_{T}\right)-w\left(0^{+}\right)=-\frac{\nu}{2 \Omega} \frac{\partial}{\partial \bar{x}} \int_{\zeta=0^{+}}^{z=h_{T}} \frac{\partial^{2} v}{\partial \bar{x}^{2}} d z
$$

Combining these two equations yields

$$
\Delta w=w\left(h_{T}\right)-w\left(-h_{B}\right)+\frac{v}{2 \Omega} \frac{\partial}{\partial \bar{x}} \int_{-h_{B}}^{h_{T}} \frac{\partial^{2} v}{\partial \bar{x}^{2}} d z
$$

Then, using (33) and (35) to eliminate $w\left(h_{T}\right)$ and $w\left(-h_{B}\right)$, equation (A 4$)$ is reduced to

$$
\Delta w=\frac{\partial}{\partial \bar{x}}\left\{-\frac{1}{2}\left(\frac{\nu}{\Omega}\right)^{\frac{1}{2}}\left[v\left(h_{T}\right)+v\left(-h_{B}\right)\right]+\frac{\nu}{2 \Omega} \int_{-h_{B}}^{h T} \frac{\partial^{2} v}{\partial \bar{x}^{2}} d z\right\}
$$

Equations (A 2), (A 3) and (A 5) can be combined to yield the form

$\Delta w^{(n)} \sec \alpha=$

$$
\frac{\partial}{\partial \bar{x}}\left\{-\frac{1}{2}\left(\frac{\nu}{\Omega}\right)^{\frac{1}{2}}\left[v\left(h_{T}\right)+v\left(-h_{B}\right)\right]+\tan \alpha \sin \theta \frac{\Delta p}{2 \Omega}+\frac{\nu}{2 \Omega} \int_{-h_{B}}^{h_{T}} \frac{\partial^{2} v}{\partial \bar{x}^{2}} d z\right\}+O\left(E^{\frac{1}{3}}\right),
$$

which can be readily integrated across the $\frac{1}{3}$-layer. The result is

$$
\begin{aligned}
& \int_{-\infty}^{\infty} \Delta w^{(n)} \sec \alpha d \bar{x}= \\
& \quad-\left.\left\{\frac{1}{2}\left(\frac{\nu}{\Omega}\right)^{\frac{1}{2}}\left[v\left(h_{T}\right)+v\left(-h_{B}\right)\right]-\tan \alpha \sin \theta \frac{\Delta p}{2 \Omega}\right\}\right|_{\bar{x}=-\infty} ^{\bar{x}=+\infty}+\left.\frac{\nu}{2 \Omega} \int_{-h_{B}}^{h_{T} T} \frac{\partial^{2} v}{\partial \bar{x}^{2}}\right|_{\bar{x}=-\infty} ^{\bar{x}=+\infty} d z+O\left(E^{3}\right) .
\end{aligned}
$$

Matching the $\frac{1}{3}$-layer solution to the geostrophic solution gives the values of the various quantities in (A 6), i.e.

$$
\begin{aligned}
\lim _{\bar{x} \rightarrow+\infty} v(\bar{x}, \theta, z) & =\lim _{r \rightarrow a} v_{\theta}(r, \theta, z), \\
\lim _{\bar{x} \rightarrow-\infty} v(\bar{x}, \theta, z) & =\lim _{r \rightarrow a} v_{g}(r, \theta, z),
\end{aligned}
$$

and similarly for the reduced pressure. Therefore, noting that $v_{g}$ and $v_{G}$ are of order 1,

$$
\left.\frac{\nu}{2 \Omega} \int_{-h_{B}}^{h_{T}} \frac{\partial^{2} v}{\partial \bar{x}^{2}}\right|_{\bar{x}=-\infty} ^{\bar{x}=+\infty} d z=O(E) .
$$


Since $v_{g}$ and $v_{G}$ are independent of $z$ to first order, necessarily

$$
-\left.\frac{1}{2}\left(\frac{\nu}{\Omega}\right)^{\frac{1}{2}}\left[v\left(h_{T}\right)+v\left(-h_{B}\right)\right]\right|_{\bar{x}=-\infty} ^{\bar{x}=+\infty}=\left(\frac{\nu}{\Omega}\right)^{\frac{1}{2}}\left[v_{g}(a, \theta)-v_{G}(a, \theta)\right]+o\left(E^{\frac{1}{2}}\right)
$$

The pressure is easily shown to be independent of $z$ outside the Taylor column to $O\left(E^{\frac{1}{2}}\right)$, so that

$$
\lim _{\bar{x} \rightarrow+\infty} \Delta p=o\left(E^{\frac{1}{2}}\right) \text {. }
$$

However, (21) shows that $p_{g}$ has a jump of order $E^{\frac{1}{2}}$ across the plate. Denoting the jump in $p_{g}$ by $\Delta p_{g}(a, \theta)$ one may write

$$
\left.\tan \alpha \sin \theta \frac{\Delta p}{2 \Omega}\right|_{\bar{x}=-\infty} ^{\bar{x}=+\infty}=-\tan \alpha \sin \theta \frac{\Delta p_{g}(a, \theta)}{2 \Omega}+o\left(E^{\frac{1}{2}}\right) .
$$

Substitution of (A 7)-(A 9) into (A 6) yields the desired relation

$$
\int_{-\infty}^{\infty} \Delta w^{(n)} \sec \alpha d \bar{x}=\left(\frac{\nu}{\Omega}\right)^{\frac{1}{2}}\left[v_{g}(a, \theta)-v_{G}(a, \theta)\right]-\tan \alpha \sin \theta \frac{\Delta p_{g}(a, \theta)}{2 \Omega}+o\left(E^{\frac{1}{2}}\right)
$$

All the terms on the right-hand side of (A 10) depend only upon the geostrophic flow variables as desired.

\section{REFERENCES}

Gremenspan, H. P. 1968 The Theory of Rotating Fluids. Cambridge University Press.

JACoBs, S. 1964 The Taylor column problem. J. Fluid Mech. 20, 581.

Moore, D. W. \& Saffman, P. G. $1969 a$ The structure of free vertical shear layers in a rotating fluid and the motion produced by a slowly rising body. Phil. Trans. Roy. Soc. A 264, 597.

Moone, D. W. \& Saffman, P. G. $1969 b$ The flow induced by the transverse motion of a thin disk in its own plane through a contained rapidly rotating viscous liquid. $J$. Fluid Mech. 39, 831.

Pendosky, J. \& Greenspan, H. P. 1967 A simple laboratory model for the oceanie circulation. J. Fluid Mech. 27, 291.

Stewartson, K. 1966 On almost rigid rotations. Part 2. J. Fluid Mech. $26,131$.

TAYLoR, G. I. 1922 The motion of a sphere in a rotating liquid. Proc. Roy. Soc. A 102, 180.

Wilcox, D. C. 1970 The motion of a disk at angle of attack in a rapidly rotating fluid.

Ph.D. thesis, California Institute of Technology. 